\title{
1D models of the thermohydraulic systems supported by CFD results and measured data
}

\author{
Václav Čibera ${ }^{1, a}$, David Lávička ${ }^{1, b}$, and Jindřich Kňourek ${ }^{1, c}$ \\ ${ }^{1}$ University of West Bohemia, New Technologies - Research Centre, Univerzitní 8, CZ 30614 Plzeň, Czech Republic
}

\begin{abstract}
In this paper is reported the way of simulation of thermohydraulic systems as whole real systems consisting of many parts. The crucial demand in our approach was to develop a software within the whole thermohydraulic system could be quickly designed and simulated under different conditions. Therefore, new graphical "drag and drop" thermohydraulic library is being developed under MATLAB/Simulink. Pivotal point in our approach was the incorporation of results obtained in CFD simulations and the incorporation of measured data from calorimetric tunnel. Designed and simulated easy cooling circuit is shown as an example of application of developed software. The results for various composition of cooling fluid and various RPM of pump are presented.
\end{abstract}

\section{Introduction}

It can be said that in the last decades the key simulation method in the fields concerning fluid mechanics and heat transfer is conducted by finite element method (FEM). Nowadays, this approach gives undoubtedly one of the best results in the actual state of art in numerical simulations. Many of CFD and heat transfer simulations were achieved in our laboratory also. Apart from CFD simulations, many of measured experiments on such parts of thermohydralic systems as heat exchangers [2] were accomplished in our laboratory too. Although a lot of desired results were obtained, the problem arose, when there was a demand on how some simulated or measured parts in system could affect another parts in the whole thermohydraulic system and how the parts behave in the whole system themselves. It was impossible to measure or simulate the whole thermohydraulic systems on account of diverse reasons. The disadvantage of FEM models is mostly a long time in preparation also as in the inflexible quick rearrangement of model usually because of geometry (re)meshing. Also the experimental measuring of the more complex thermohydraulic systems was found to be much complicated because of the reasons such as inflexible rearrangement of components in system etc.

Although for aim of this paper the results obtained in FEM solvers and measured data are crucial, the main target of this contribution is to show how to avoid long computation by reusing the obtained results. In this paper is reported the progress in the development of software based on the 1-D mathematical models already introduced in [1]. In our approach was the main goal to quickly simulate the whole thermohydraulic systems with different conditions such as various number of parts in system, different composition of cooling fluid, different rotation per minute (RPM) on pump, different heat transfer efficiency on radiator, different load of engine etc.

\footnotetext{
a e-mail: vacibera@ntc.zcu.cz

b e-mail: dlavicka@ntc.zcu.cz

c e-mail: knourek@ntc.zcu.cz
}

Currently, the first part of work was devoted to improvement of cooling fluid properties settings. Because the cooling fluid composition also plays a key role in heat transfer through the system and strongly impacts the pressure drops across the system, the main task was to incorporate the properties of the cooling fluid to the presented software and simulations. The second part of work was concerned with integration of the pump properties into the cooling circuit.

The article is set out as follows: the first part of contribution is introduction. In the second section follows description of used methods and presentation of basic properties of developed software Thermohydraulic NTC. In the third section is presented easy design of thermohydraulic system using developed library. This section also includes the results of two simulations, where in the first simulation was investigated volume flow rate, $l / \mathrm{min}$, of coolant through system depending on various cooling fluid composition. In the second case was investigated volume flow rate, $l / \mathrm{min}$, of coolant through the system with respect on RPM of pump. The paper end up conventionally with last section containing conclusion discussing advantages, disadvantages and limits of chosen approach.

\section{Description of methods}

As far as thermohydraulic systems are concerned in presented paper in general, seven crucial physical variables were taken into account to characterize problem sufficiently: volume flow rate, $l / \mathrm{min}$; temperature, $K$ or ${ }^{\circ} \mathrm{C}$; heat flow through components, $W$; pressure drops across elements, $\mathrm{Pa}$; and three variables attributed to describe the basic properties of cooling fluid: density $\mathrm{kg} \mathrm{m}^{-3}$; kinematic viscosity, $\mathrm{m}^{2} \mathrm{~s}^{-1}$, and specific heat capacity, $J \mathrm{~kg}^{-1} K^{-1}$. Apart from these physical variables, another one time variable and three space variables are needed to depict the system sufficiently. This number of variables leads to the mathematical model consisting of partial differential equations that is exactly what makes the simulation more time consuming because of numerical solution. In addition, when 
there is a demand on system rearrangement i.e. change of spatial distribution of system parts, in the case of solving by FEM for example, the re-meshing of the whole model must be usually performed.

To avoid obstacles noted above, it was decided to try to use 1-D mathematical models, where only one spatial variable is taken into account. The main aim was to reduce the mathematical model represented by partial differential equation to mathematical model consisting only of ordinary differential equations or algebraic-differential equations. This simplification was carried out under assumption that almost each of flows as a heat flow or fluid flow in our systems is supposed to flow only in one concrete direction. It was also assumed that the heat is transferred just in specific parts or boundaries in system. Therefore, in vast majority of our problems, the description could be substituted by 1-D mathematical models.

Because this simplification was already studied many times probably for the same reason as in this contribution, considerable number of 1-D mathematical models describing fluid flow through the pipes and heat flow can be found in literature. In the best case, these 1-D models are by itself physical laws and in other case these 1-D mathematical models originate from empiric observation. For this paper the striking features which distinguish among empiric formulas and formulas expressed as general physical laws is the range of validity. Let's see for instance the first law of thermodynamics describing preservation energy. It is no doubt that this must be valid at every scale and also for processes taking place at every rate. In opposite to this, let's see for example Coolenbrook-White formula describing pressure looses with respect on flows in pipes. Although it is widely use, its limitation is that it is valid only for certain range of Reynolds numbers that is for Reynolds number expressing turbulent flow. Another 1-D mathematical models must be used for the cases of the different kinds of flows. Though these models also can be found, the crucial problem in our work arose from the fact that this feature of empirical laws can make the mathematical model discontinuous. Since then there could be some restriction on ordinary differential equation solver.

Except the 1-D mathematical models, the second crucial way in approach was the reuse of computed or measured characteristic properties of specific parts in thermohydraulic systems. It must be pointed out here that these characteristics are usually computed by CFD hence, as it was already mentioned, FEM results plays also crucial role for model preparation. If these characteristic properties or data are obtained for sufficient wide range of validity, they can be used to derive mathematical formula from them in ideal case. Another case is also interpolation from these data. Both of these approaches were used in presented work.

The last step was to chose the tools to make the model more well arranged. Although a lot of suitable software already exist, it seemed to be better to develop own software. The main reason for this decision arose mainly from the fact that it would be easier to include computed and measured data into our models. As the most feasible way to achieve our goals it seemed to chose as a working environment MATLAB with Simulink. It was selected due to the fact that it is well-known among the engineers and scientists. This development environment also offers a lot of already prepared tools, which makes the work much easier.
Besides computational tools, it also offers tools to develop own drag and drop graphical library within Simulink. There is more than one prepared tools to achieve this. It was decided to develop new drag and drop library Thermohydraulic NTC [1] using Level-2 MATLAB S-Function [4]. In brief, by application of these tool can be developed graphical blocks representing mathematical functions. In this works, the graphical blocks are though to represent real parts of thermohydraulic system. Every part of system, i.e. graphical block, is characterised by its own set of equations and data. This settings then allows change of input signal to this block according to figure 1. Signal here contains information about physical variables noted above from the preceding parts of system. For example the input temperature is changed according to inlet heat on component and outlet temperature has new value, which impacts next component representing another part of system. This

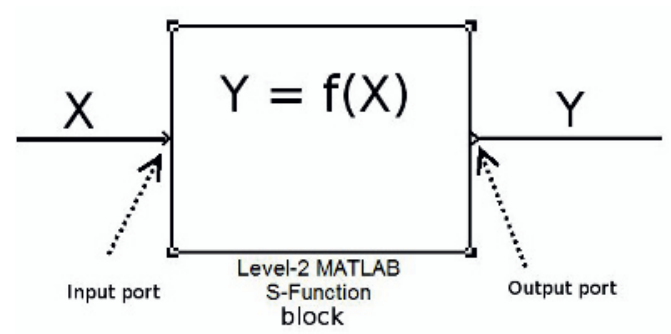

Figure 1: Graphical block signal approach scheme

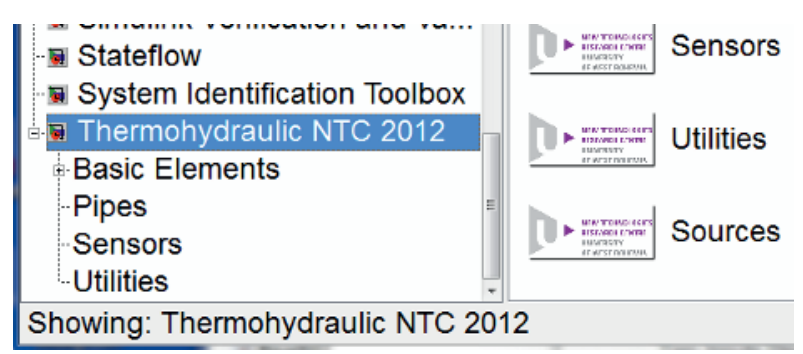

Figure 2: Thermohydraulic library incorporated in Simulink

way was developed new graphical drag and drop library which is incorporated into Simulink (figure 2 ). Library contains mainly components for simulation of cooling circuits in cars. So there can be found pipes, heat exchangers, pump etc. Every of graphical blocks, which represent a real part of system, serves also as an ideal sensor. Easy doubleclick opens graph depicting each of every seven variables stated above during the time (figure 3).

On the pipes can be observed also another properties as Reynolds number or pressure drops in the system also as pressure drop on specific part of system. Double-click on graphical blocks also opens settings of various properties. For instance, last work was focused mainly on incorporation of cooling fluid properties and its easy changes to the simulation. Special graphical block of cooling properties was developed then and as an example it is possible to change proportion ethylene-glycol in cooling medium consisting of ethylene-glycol and distilled water (figure 4). 


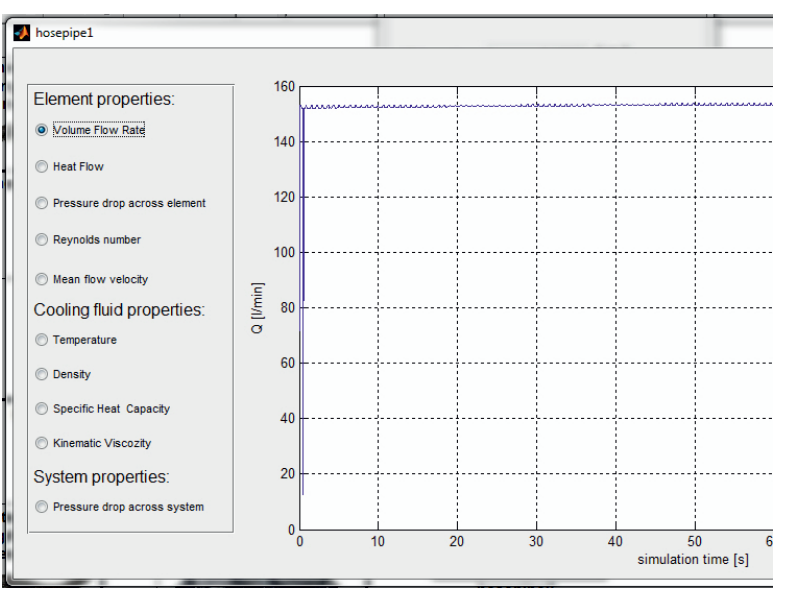

Figure 3: Pipes serves also as an ideal sensors in system

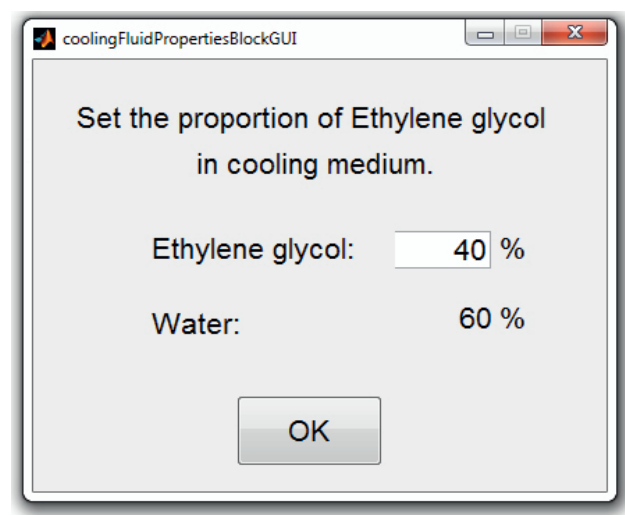

Figure 4: Cooling fluid set-up

\section{Simulation and results of simulation}

First, basic unbranched circuit was assembled of elementary parts such as: pump, pipes, engine (heat source), heat exchanger and naturally of cooling medium. Designed cooling circuit is shown in figure 5. The mathematical background of pipes is well described by 1-D mathematical models. In the pump are used computed data by CFD simulations obtained in our laboratory. Data describing behaviour of heat exchanger was measured in calorimetric tunnel in our laboratory. The deficiency in our simulation model is behaviour of engine. This imperfection is due to the fact that there were no characteristic data computed or measured till now. Therefore the graphical block representing engine in our model contains only estimated data. This part could be then considered just as an element with rigid hydraulic resistance and with constant heat transferred into the cooling fluid. After the simulation model was set up, two simulations were conducted.

\subsection{Simulation with various cooling fluid properties}

As a first example was simulated cooling circuit with various composition of cooling medium. It was assumed that as cooling medium in cooling circuit was used liquid consisting of mix distilled water and ethylene-glycol. Three simulations we computed for three different composition of cooling fluid. Proportion of ethylene-glycol in mixture

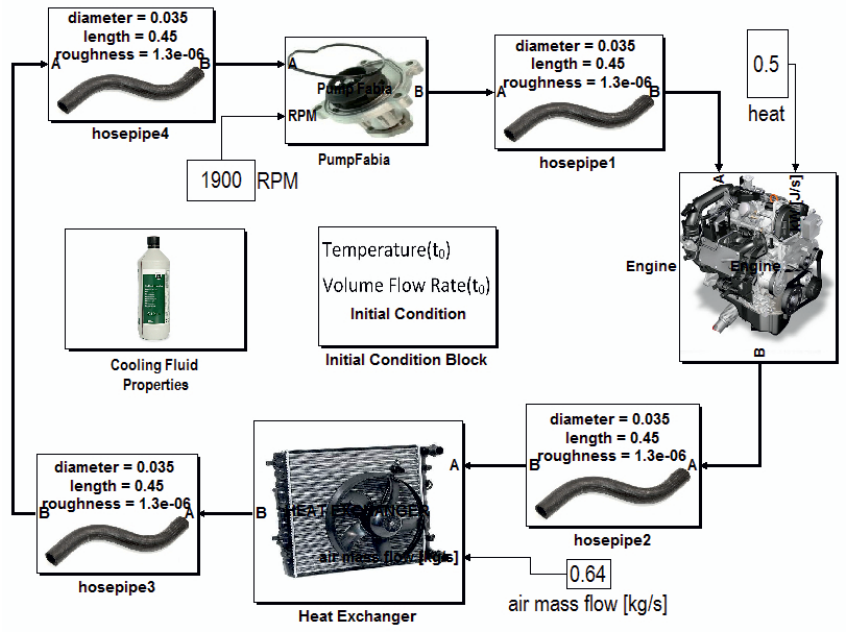

Figure 5: Testing circuit

was set to values $10 \%, 40 \%$ and $90 \%$. Only this property of cooling circuit was changed. The simulation was executed for constant RPM of pump that was 1800 RPM and simulation time was set to 30 seconds.

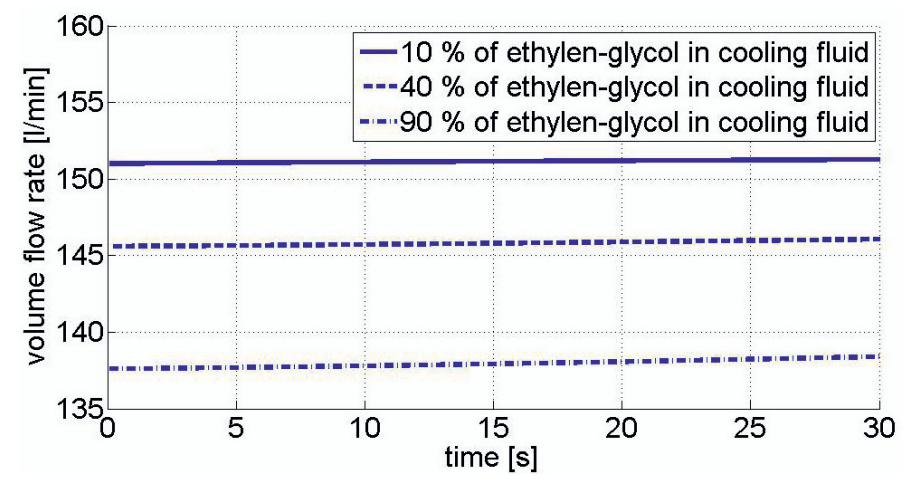

Figure 6: Impact of the coolant composition on coolant volume flow rate through system by 1800 RPM of pump

The results of these three simulations are depicted on the graph in figure 6 . The highest values for volume flow rate through system was obtained for cooling fluid composition consisting of $10 \%$ ethylene-glycol in compound and the less values of volume flow rate through system was obtained for cooling fluid consisting of $90 \%$ of ethyleneglycol in compound. Comparing these results makes sense according to fact that the cooling fluid with higher proportion of ethylene-glycol in mixture with distilled water form cooling fluid with higher value of kinematic viscosity. By implication of higher kinematic viscosity, there were higher pressure drops in system. Therefore the pump had to overcome higher pressure difference across itself and it consequently resulted in lesser volume flow rate though the system, which is in concordance with observation.

As can be seen on the graph in figure 6, the curves describing volume flow rate through the system were not constant. During simulation time, the volume flow rate raised for each of mixture composition. Owing to the fact that the system was heated on graphical block representing engine, the cooling fluid raised in temperature. It consequently implicated change in cooling fluid viscosity. Cooling medium 
became less viscous and resisted with lesser hydraulic resistivity which resulted in enhanced volume flow rate. The average volume flow rate for $10 \%$ mixture was $150.99 \mathrm{l} / \mathrm{min}$, for $40 \%$ mixture was $145.88 \mathrm{l} / \mathrm{min}$ and for $90 \%$ of ethylene-glycol in mixture the average volume flow rate was $137.97 \mathrm{l} / \mathrm{min}$. The difference between mixture with $90 \%$ and $10 \%$ proportion of ethylene-glycol in mixture is about $8.6 \%$. It is arguable if this amount can affect cooling ability of the system in normal conditions, but in some extreme conditions this difference can influence the behaviour of system.

\subsection{Simulation with various value of RPM}

As the second example was simulated impact of the change of pump RPM on the volume flow rate through the system. As already mentioned above, the description of pump behaviour is based on the obtained data from CFD results. A wide range of data describing flows depending on pressure losses is incorporated to pump properties. On account of pressure losses in cooling circuit during the simulation, the pump generated volume flows trough the system. The simulations were conducted for five RPM: $1000,1500,1800,2200,2500$. The composition of the cooling fluid was $40 \%$ of ethylene-glycol and $60 \%$ of distilled water. The simulation was conducted for 50 seconds. The results are shown in figure 7 . As expected, the highest value

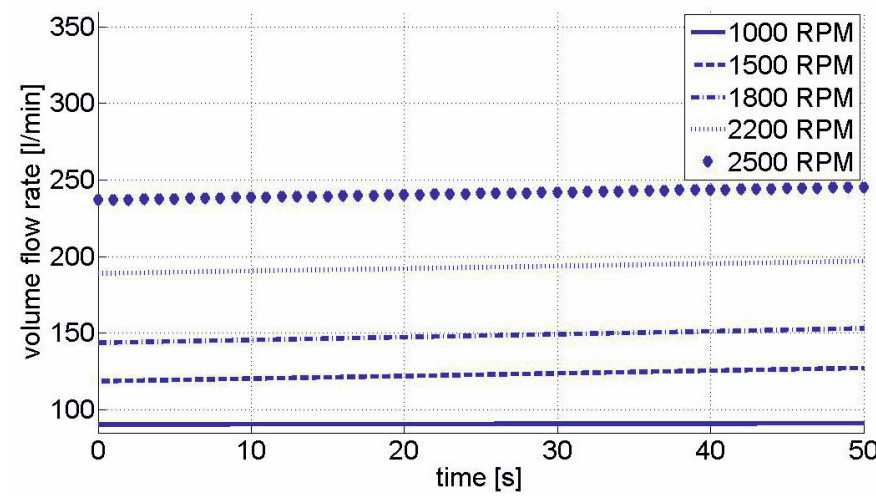

Figure 7: Volume flow rate $l / \min$ for various rotation per minute of pump

for volume flow rate through system was obtained for highest RPM of pump. In opposite, the smallest value was obtained for the smallest RPM of pump. The main purpose of these computation was mainly to try if this way of computation and data processing is even possible. Again, it can be seen that the curves describing volume flow rate through system arose because of the same reason as pointed already above. This also assured us that this way of data processing is useful and the properties of pump simulated like this could give basic idea about the behaviour of pump in system consisting of many parts.

\section{Conclusion}

In this contribution was presented developed software integrated into MATLAB/Simulink environment. This software or better thermohydraulic library Thermohydraulic
NTC was designed for easy and quick simulation of thermohydraulic systems. The main purpose of the work was to reuse already computed (CFD) and measured (calorimetric tunnel) data. Adapted data was integrated into the 1-D mathematical models based on physical or empirical laws. Based on these mathematical models was developed and is further being developed drag and drop graphical library under Simulink. Using simplification and methods noted above, requirements on hardware are not hight and the computation is not so time-consuming as FEM computation, though the characteristic behaviour of many parts of systems had to be achieved by FEM computation in advance. Using this software, it is possible to get basic idea about thermohydraulic systems behaviour in the range of minutes. Setting new properties and easy rearrangement of system topology can be done also in the range of minutes.

Two kind of simulations were presented. As a first example was simulated impact of the cooling fluid compound on the volume flow rate through the system. As a second example was tested computed data processing for use in pump. All of the results corresponded to expected behaviour of thermohydraulic systems. In the first simulation was examined the impact of the cooling fluid composition on the volume flow rate through the system. As expected, the highest value of volume flow rate was obtained for composition with less kinematic viscosity and vice versa. The main purpose of the second simulation was to try incorporation of computed data from CFD results into dynamical system simulation. Characteristic data were applied into 1-D mathematical models. Consequently, the simulation was conducted for various RPM. According to the expected results, the slowest values of volume flow rate were obtained for the smallest values of RPM and vice versa.

The results assured us and indicated that this way of investigation can be found very useful and give basic description about behaviour of thermohydraulic system parts such a pump in circuit in short time. Still a few challenges remain for another work. It is needed to concentrate more properly on the setting of initial conditions of mathematical model and numerical solution of this model, because some results were not smooth enough at the beginning of simulations. Although the drag and drop library is still being developed, it is able already now to give attractive output results, which are consistent with desirable behaviour of investigated systems. As a great advantage seems to be fact that the library was integrated into the Simulink, where also another tools from Simulink sub libraries can be used.

\section{Acknowledgement}

The work is supported by grant SGS-2012-072.

\section{References}

1. V. Cibera, D. Lavicka, Exp. Fluid Mech., 45, 123-126 (2012)

2. J. Knourek, M. Kus, T. Syka, Exp. Fluid Mech., 25, 754 - 757 (2011)

3. T. Syka, M. Kus., Exp. Fluid Mech., 648 - 655 (2010)

4. MATLAB version R2012 User guide, help, The MathWorks, Inc., Natick, Massachusetts, United States. 